\title{
Building blocks of competitive intelligence - competitive technical intelligence
}

\author{
Marié-Luce Muller \\ Director and Analyst \\ IBIS Business and Information Services \\ mlm@ibis.co.za \\ www.ibis.co.za
}

\section{Introduction}

In the previous column, I wrote that competitive intelligence (CI) is not a substitute for other business processes, but rather that it is comprised of a number of building blocks that hopefully integrate information obtained from these processes to create a comprehensive, interpretable picture of a company's possible future competitive environment at any given time. CI comprises many aspects of which the following are utilized most often: competitor intelligence, technical intelligence, market and marketing intelligence, financial intelligence, product intelligence and customer intelligence.

In this column, the focus is on competitive technical intelligence (CTI).

\section{Defining CTI}

CTI can be regarded as more of a sub-set of CI than a building block. The main distinguishing factor is that there is a shift more to a technological than a business focus.

In defining CTI, a number of key words arise including competitiveness, better decisionmaking, technical environment and continuous systematic process. The Society of Competitive Intelligence Professionals (SCIP 2006) defines CTI as 'the process focused on monitoring the competitive and technical environment of an organization (company, university, entity for the purpose of better decision making by high and middle level executives in the areas of marketing, product design, research and development (R\&D), which can be used from investment tactics to long-term business strategies'. It is a form of competitive intelligence.

Bryant (1998) states that CI involved two information tracks namely competitive information derived from technical or business data. The choice of technical or business data and the analyst's background will determine whether the results are CTI or competitive business intelligence. Combining the two will lead to better business decisions.

CTI comprises a continuous and systematic process that implies the legal and ethical collection of information, analysis and production of actionable results for the strategic planning process and controlled diffusion of main findings. Ashton (1997) defines CTI as actionable information about external science and technology (S\&T) developments and trends that could impact on a company's competitive position. He emphasizes the importance 
of analytical findings about the S\&T environment.

\section{Importance and focus of CTI}

Having the right technology at the right time is becoming an increasingly important part of business success. In this fast changing business environment, companies, especially those in high technology and fast moving industries, need accurate and timely intelligence on their technical environment if they expect to manage their technical resources wisely.

Technical CI is important in all industries, not only in science-based companies such as pharmaceuticals and chemicals, and has an impact on all steps of the operation, from research and development (R\&D) through manufacturing to marketing and sales. CTI distinguishes itself by the need to analyse and interpret scientific and technical information, which requires a basic understanding of science and technology to be successfully implemented. The approach and thinking process required is different from most general CI questions. The technical CI process is not simply a cycle of researching the literature, gathering information and communicating the findings. At first glance, the issues to be addressed in technical CI often appear to be very specific. But a broader knowledge of the technology or science is required to answer the real CI questions (Hefti 2003).

CTI focuses mainly on technological threats, opportunities and developments and is typically more prevalent in industries such as aerospace, biotechnical, chemical and consumer products, energy, information technology, manufacturing, pharmaceuticals, telecommunications and transportation. These industries rely on innovativeness to provide a competitive edge, and maintaining innovativeness is an ongoing challenge for companies. Typically, companies in the biochemical industry rely on CTI to put in place systems that alert them to emerging breakthroughs in research laboratories throughout the world, and to the future possibilities that these advances provide (Miller 2000).

Technical CI is not limited to the R\&D division of a company; it impacts most other business operations. Properly gathered and interpreted, and combined with insights from the technical CI professional, it provides important warning signals about a competitor's operational plans. The planned (but not yet publicized) introduction of new products, changes in R\&D plans and other similar activity is visible to people who can review and dissect the related processes (Hefti 2003).

\section{Benefits and purpose of CTI}

Some of the benefits of a systematic CTI capability include continuous monitoring of competitive environments to enable early detection of opportunities for technical innovation, including the design of new processes and products, informed decisions, information converted into intelligence (results tailored to decision making needs), identification of new areas of research and mapping structural processes of a particular area of research.

CTI should provide clarity to company decision makers as to actions that will lead to opportunities to defend, grow or improve competitiveness or that will create business assets (Ashton and Klavans 1997). Ashton and Klavans describe CTI as a function that creates forward thinking and planning and serves as the 'motor for ideas or the non-physical assets of a company'.

CTI helps companies to develop, manage and use their own technical intelligence programmes to gain a competitive advantage. Having the right technology is a part of a business's success. Because of the constantly changing and competitive economy, a company needs accurate and timely intelligence if it expects to manage its technical resources wisely. 
CTI activities play an important role in strategic decision-making by providing early warnings and focusing long-term goals into shorter-term tactical objectives. Clear objectives are the core of successful CTI and its ability to advise R\&D. In contrast to CI where literature has recommended a certain level of autonomy in organization CI structure, CTI recommends a close alliance to $\mathrm{R} \& \mathrm{D}$ (not marketing and sales).

CTI could prevent nasty surprises and could alert companies in time to opportunities and threats arising from emerging technologies that could be critical to their competitive position so they can be seized in advance of the competition. It can also ensure that companies stay abreast of changes in markets, customers, technologies and competitors. Technical intelligence analyses and foresees changes in competitors' technologies and business strategies, trends in the industry as a whole, as well as changing expectations among markets and customers. CTI provides an understanding of how the various bits of technical and market information fit together and what they mean for a company.

It should also provide companies with the ability to quickly frame, monitor and coordinate possible business opportunities and not lose focus on internal opportunities. It could promote idea development and internal collaborations within the company, fostering ties with researchers who might otherwise leave to form their own start-up companies.

\section{Key to good CTI programmes}

- Flexible, easily adjustable programmes

- Maintaining excellent relations and open communication with stakeholders

- Clear communication of stakeholder objectives across management teams and the company

- Capital and resources to be committed to CTI specifically and not just broadly to a department or to marketing research

- Across all successful CI programmes, management recognized different functions within CI and also addressed CI as a process supported by IT but not as a function of IT, that is, only data mining, database management, etc (Lackman, Saban and Lanasa 2000)

- Technical intelligence is usually an ongoing operation, keeping decision-makers up to date with all the critical factors that affect their business.

\section{Case study in CTI}

At SCIP's CTI Symposium in 2000, Tim Budd, director of business development at Applied Biosystems (formerly PE Biosystems), in his keynote address described how his firm, a leading biotechnical innovator and the second-largest employer of high-technology people in the Bay Area, had put in place systems that alerted it to emerging breakthroughs in research laboratories throughout the world and to the future possibilities that these advances provided. Herewith a few highlights of his address (Miller 2000):

\section{Reasons for performing CTI}

- Need to achieve and sustain innovativeness

- Need to focus beyond the product of today, or even the innovations that customers want tomorrow, on what is going to drive sales in three to five years' time

- Need to create 'an idea incubator'

- Need to continually develop new products and technologies that exploit advances that are not easily perceivable today.

\section{How they do it}


- Established an independent S\&T exploratory group, responsible for looking at longterm opportunities rather than short-term product development

- The S\&T group scouts for totally new, often disruptive new technologies, as opposed to the next incremental improvement, but with the responsibility of selling those to the business units to develop

- The S\&T group is comprised both of researchers and business types who rotate in and out from other units including outside laboratories that partner with the companies, but it receives its own research budget annually

- The group enters into partnerships and external alliances with researchers at leading private, university and government laboratories conducting research at the prototype stage

- Alliances are also made with large companies. Decisions many companies face include whether to continue to invest in new hard assets, whether to build new manufacturing plants or whether there is a potential new partnership. A reason for such alliances could be to utilize high-volume manufacturing capabilities or share technologies, for example, automotive manufacturers often collaborate on technological developments

- The group strives for systematic and continued interaction with internal project teams, working together and rotating people from the operating units into the S\&T group when they want to 'tackle something new' (Miller 2000).

\section{Tools and skills for CTI}

Hefti (2003) mentions that CTI professionals should be familiar both with the analytical tools suitable for technical competitive intelligence (CI) and how it differs from a more general approach to CI.

A CTI programme officer, for example, should typically pursue development of the CTI programme by providing input to the programme's strategic direction and product development, leading the contracting process for CTI service delivery with internal stakeholders and external clients, monitoring the quality and effectiveness of CTI services, supporting implementation of CTI services within research institutes and programmes and promoting CTI within the company.

Insights acquired over time and with experience are essential for monitoring research activities. The technical CI professional has to be able to identify new technologies and assess their potential value. Emerging technologies may provide a threat or an opportunity, depending on the company's ongoing activities and business interests. This should of course always be translated into a business opportunity to benefit the company. A strong business background is therefore advisable.

To recognize the warning signals, the technical CI professional has to think through the entire process and identify relevant activity and changes. The technical CI professional may not have all of the specialized knowledge or experience required, but he or she needs to know when experts are required to complete the picture.

A technical CI professional has to be adaptable to change; there are rarely two situations where the same approach leads to results. A broad science knowledge base is required, supplemented by a sense of business to fit the science into a business case.

\section{CTI process}

CTI utilizes the well-known CI process with the difference being that the focus is shifted to technical more than to business. 
- Planning and focus for a needs assessment and defining key intelligence topics. CI should only focus on those business issues that are of critical importance for a company to know. These issues are known as key intelligence needs or requirements. Typical topics could include tracking new knowledge, potential new players and potential new technological breakthroughs, assessing current technology use, emerging technology processes, potential disruptions and new approaches and applications, and assessing new business prospects, market niches and key players' relations. A key decision maker might ask whether there are competing or complementary technologies, who the competitors are, what role technology plays in their competitive advantage and what the potential applications for a new technology are.

- Collection (search and validate). It is during this phase that information is collected from a variety of sources for examination and verification during the CI process. Collection comes from a variety of different sources and gathering techniques. A variety of internal, external, primary (verbal) and secondary (published, written) sources should be used. Internal sources could include information specialists, legal and patent staff, and specialists in the company, for example technology forecasters and R\&D staff. External sources should include technical colleagues, industry experts and analysts, university staff and consultants. There are a myriad of secondary sources one could access, for example internal databases, R\&D plans, project proposals, published research and market assessments, patent layers, databases, annual reports and science publications.

In technical CI, information is rarely available directly from the companies that are being targeted. The CI professional needs to create a network of sources interacting with the target company, including suppliers of services and goods, diverse government agencies and customers. This network can help provide the elusive pieces of data and information. In addition, network analysis can reveal experts who will be helpful in interpreting the findings.

- Analysis (interpret and report). During this phase, information is turned into intelligence through a process of interpretation and the results should be useable in strategic decision-making.

CTI is most valuable to guide and support various business decisions. Any due diligence analysis includes a competitive assessment of the company's operations. To understand the competitor's ongoing activities and plans, diverse signals have to be reviewed and interpreted to provide clues about $R \& D$, manufacturing and product rollout approaches. For example, a specific company's new research collaborations and scientist hiring can shed light on likely development priorities.

To leapfrog the competition, a visionary path from the future to the present has to be built. Technical analysis and scenario review play a major role in this process. The technical CI professional has to be able to project beyond the printed patent information to interpret plausible knowledge.

Patent analysis and scenario play can help evaluate options and lead to realistic conclusions. Other popular analysis tools mentioned by Ashton (2004) include scenario analysis, cost analysis (product, process and technology), trend analysis and technological assessments, and the popular Porter Five Forces, strengths, weaknesses, opportunities and threats (SWOT) analysis and competitor/persons profiling.

- Communication (presentation and delivery). The results of the CI process are communicated to those with the authority and responsibility to act on the findings in an appropriate format and at the right time.

Technical intelligence can be difficult to communicate to decisionmakers because they might not know all the technical points that impact a decision. Also, technical knowledge is specialized and unfamiliar to many executives who deal primarily with financial and market data. Often the CI professional has to explain and justify the new and different approach proposed and the message should not be conveyed in a complicated manner.

Timing is critical for technical intelligence reports. Technical intelligence is typically 
fast moving and change can occur quickly. A lengthy report defeats its purpose; a visual interpretation can achieve more. The actual form of communication (oral presentation, written report, tables) should be tailored to the recipient's preferences. A technical CI report has to include recommendations. It is up to the decision maker to accept or dismiss them, but presenting just data is not enough. Translating technical knowledge into business terms requires taking a stand and predicting likely outcomes. The insight provided by an experienced CI professional should facilitate executive decision (Hefti 2003).

In summary, the communication kit for CTI would typically include alerts, technical newsletters, ad hoc reports and also more specialized reports such as comprehensive assessments and strategic forecasts.

- Process and structure. CI requires appropriate policies, procedures and an infrastructure so that employees may contribute effectively to the CI system as well as gain the benefits from the CI process.

- Organizational awareness and culture. For a company to utilize its CI efforts successfully, an appropriate organizational awareness of CI and a culture of competitiveness are necessary. While decision makers should call the shorts on what intelligence is required, information gathering should be on everyone's mind (Kahaner 1997).

\section{References}

Ashton, W.B. and Klavans, R.A. 1997. Keeping abreast of science and technology: Technical Intelligence for business. Columbus.

Ashton, B. and Archambeault, J. 2004. Competitive technical intelligence: an introductory workshop. Canada: Halifax.

Hefti, E. 2003. Technical analysis and skills set. Competitive Intelligence Magazine 6(6).

Kahaner, L. 1997. Competitive intelligence. How to gather, analyse and use information to move your business to the top. New York: Simon and Schuster.

Lackman, C.L., Saban, K. and Lanasa, J.M. 2000. Organising the competitive intelligence function: a benchmarking stuffy. Competitive Intelligence Review 11(1).

Miller, S. 2000. SCIP's competitive technical intelligence symposium. Competitive Intelligence Magazine 3(4).

Muller, M.L. 2004. Key intelligence needs: the roadmap of your competitive intelligence capability and activities. South African Journal for Information Management 6(1).

SCIP, 2006. [Online]. Available at https://www.123signup.com/servlet/SignUpMember? $\underline{P G=1857182300 \& P=1857191153067700 \& I n f o}$. (Accessed on 20 July 2006).

\section{About the author:}

Marié-Luce Muller is a consulting CI analyst with IBIS Business and Information Services (Pty) Ltd, a leading Pretoria-based CI consultancy. She has a distinguished career in CI. Her primary experience lies in assisting companies in honing their CI capabilities. She also performs tracking and scanning activities on behalf of companies. Marié-Luce has published many articles on competitive intelligence (CEO Magazine, Finance Week, Business Week, Beeld, Die Burger and the South African Journal of Business Management), including an article on South Africa as an emerging CI player, which was published in an international 
publication of the Society of Competitive Intelligence Professionals (SCIP). She has also published a series of booklets on CI (Nuts and Bolts business series, published by Knowledge Resources) and is a member of a research team participating in an international study of CI practices among exporting companies. Previously, she was involved in research into the status of CI practices in South Africa. A member of SCIP, she holds a postgraduate degree from the University of Stellenbosch.

\section{Disclaimer}

Articles published in SAJIM are the opinions of the authors and do not necessarily reflect the opinion of the Editor, Board, Publisher, Webmaster or the Rand Afrikaans University. The user hereby waives any claim he/she/they may have or acquire against the publisher, its suppliers, licensees and sub licensees and indemnifies all said persons from any claims, lawsuits, proceedings, costs, special, incidental, consequential or indirect damages, including damages for loss of profits, loss of business or downtime arising out of or relating to the user's use of the Website.

ISSN 1560-683X

Published by InterWord Communications for Department of Information and Knowledge Management, University of Johannesburg 